\title{
Erratum to: Principles of carbon nanotube dielectrophoresis
}

\author{
Wenshan $\mathrm{Li}^{1,3, \dagger}$, Frank Hennrich ${ }^{1,2,4}$, Benjamin S. Flavel ${ }^{1}$, Simone Dehm ${ }^{1}$, Manfred Kappes ${ }^{1,2}$, and Ralph \\ Krupke $^{1,3,4}(\bowtie)$ \\ ${ }^{1}$ Institute of Nanotechnology, Karlsruhe Institute of Technology, Karlsruhe 76021, Germany \\ ${ }^{2}$ Institute of Physical Chemistry, Karlsruhe Institute of Technology, Karlsruhe 76021, Germany \\ ${ }^{3}$ Institute of Materials Science, Technische Universität Darmstadt, Darmstadt 64287, Germany \\ ${ }^{4}$ Institute for Quantum Materials and Technologies, Karlsruhe Institute of Technology, Karlsruhe 76021, Germany \\ ${ }^{\dagger}$ Present address: School of Mechanical Engineering, Shanghai Jiao Tong University, No. 800 Dongchuan Road, Shanghai 200240, China
}

(C) The Author(s) 2021

\section{Erratum to}

Nano Research

https://doi.org/10.1007/s12274-020-3183-0

The article "Principles of carbon nanotube dielectrophoresis" written by Wenshan Li, Frank Hennrich, Benjamin S. Flavel, Simone Dehm, Manfred Kappes, and Ralph Krupke, was erroneously originally published electronically on the publisher' internet portal (currently SpringerLink) without Open Access. The copyright of the article should be changed to (c) The Author(s) 2021 and the article is forthwith distributed under the terms of the Creative Commons Attribution 4.0 International
License (http://creativecommons.org/licenses/by/4.0/), which permits use, duplication, adaptation, distribution and reproduction in any medium or format, as long as you give appropriate credit to the original author(s) and the source, provide a link to the Creative Commons license, and indicate if changes were made.

The original article has been corrected.

The online version of the original article can be found at

https://doi.org/10.1007/s12274-020-3183-0 